\title{
Magnetohydrodynamic Control of Hypersonic Separation Flows
}

\author{
Shichao Luo $\mathbb{D},{ }^{1,2}$ Jun Liu $\mathbb{D}^{1,2}$ Hao Jiang, ${ }^{1,2}$ and Junyuan Wang ${ }^{1,2}$ \\ ${ }^{1}$ College of Aerospace Science and Engineering, National University of Defense Technology, Changsha, Hunan 410073, China \\ ${ }^{2}$ Science and Technology on Scramjet Laboratory, Changsha, Hunan 410073, China
}

Correspondence should be addressed to Jun Liu; liujun@nudt.edu.cn

Received 3 November 2020; Revised 12 December 2020; Accepted 28 December 2020; Published 13 January 2021

Academic Editor: Jose Carlos Páscoa

Copyright (C) 2021 Shichao Luo et al. This is an open access article distributed under the Creative Commons Attribution License, which permits unrestricted use, distribution, and reproduction in any medium, provided the original work is properly cited.

\begin{abstract}
Magnetohydrodynamic (MHD) control of hypersonic laminar separation flows is investigated in this paper. A series of numerical simulations over various geometry configurations, namely, a compression corner and a double wedge ramp hypersonic inlet, have been conducted by application of an external electromagnetic field. Results show that the performance of MHD separation flow control is mainly determined by flow acceleration of the Lorentz force directed in the streamwise direction. The Joule heating term always brings negative effects on the MHD separation flow control and increased the static pressure locally, where the electromagnetic field is applied. With an external electromagnetic field applied, the low velocity fluid in the boundary layer can be accelerated. Moreover, there exists a best location for the MHD zone to be applied and completely eliminate the separation of the flow from the surface.
\end{abstract}

\section{Introduction}

Some of the most serious and challenging problems encountered by the designers of hypersonic vehicles arise because of the severity of the heating loads and the steepness of the flow gradients that are generated in shock wave boundary layer interaction (SWBLI) regions. Hypersonic vehicles tend to fly at high altitudes so that the characteristic Reynolds numbers tend to be low, and many practical hypersonic flows are laminar. Moreover, it may well be the laminar viscous inviscid interaction and flow separations that are associated with these flows at greater altitudes that pose the greatest fundamental limitations on the performance of maneuvering and air-breathing hypersonic vehicles [1]. The effectiveness of intakes and flap-control systems, as well as the performance of vehicles using jet interaction, may be compromised seriously by the occurrence of shock-induced laminar separation.

Because it is often difficult to avoid detrimental SWBLI occurring within a flow, the idea soon arose during the early history of the development of compressible fluid mechanics of controlling the phenomenon by an appropriate manipulation of the flow. The target of the control techniques was mainly to prevent shock-induced separation when it occurred in naturally unsteady configurations. The cause of the boundary layer separation is the adverse pressure gradient and a loss of the momentum by the friction. Therefore, if the momentum of the fluid near the wall is recovered, the boundary layer separation can be eliminated. Boundary layer separation control can be achieved using many different approaches, either before or during the interaction process, all of which modify the flow near the surface [2]. In fact, recent developments in artificial ionization techniques and improvements in superconducting materials have resulted in consideration of the electromagnetic field as a tool for modifying SWBLI in the hypersonic flow regime $[3,4]$. This flow control system has so many advantages $[5,6]$ in that it is reusable, easy to be switched on-off, highly reliable, and does no harm to the aerodynamic configuration of the object aircraft. All of these advantages have warranted popular interest in this subject area in recent years.

In the recent decades, there have been several investigations concerning the MHD control techniques for separation prevention in SWBLIs. The possibility of controlling the boundary layer separation at the hypersonic plasma flow field has been demonstrated both numerically and experimentally. As in the investigations by Fujino and Shimosawa [7], the investigations of flows over blunt bodies have demonstrated 
the beneficial effect of the applied magnetic field. In addition, many fundamental features of MHD flows over the compression corner have been revealed in works of Dietiker and Hoffmann [8]. In their CFD computations, a substantial reduction in the skin friction could be achieved by an appropriate combination of electromagnetic fields. Furthermore, the effects of a body force, called Lorentz force, which can be used to control SWBLI flow have been investigated in Ref. [9, 10]. Cheng et al. [11] researched the effect of an applied magnetic field by assuming absence of Joule heating and a constant electrical conductivity of $100 \mathrm{mho} / \mathrm{m}$ over a flat plate at Mach 4.5; the imposed magnetic fields significantly decelerated the boundary layer. Khan et al. [12] investigated the validity of a low magnetic Reynolds number approach for modeling some magneto-fluid dynamics problems. The results obtained from low magnetic number approximation compare well with the results obtained by solving the full MFD equations for low ranges of the magnetic Reynolds number $\left(R_{m} \ll 1.0\right)$. In Ref. [13], the effects of magnetic interaction parameter and load parameter on hypersonic corner shock wave boundary layer interaction flow have been investigated extensively. As to the aspect of experimental works, the theoretical experiment of MHD separation flow control system is conducted in the plasma wind tunnel by Webb et al. $[14,15]$. The feasibility of MHD separation control around a two-dimensional bump in a hypersonic air flow has been experimentally validated by Zhang et al. [16]. Experimental results show the ability of controlling the SWBLI configuration at a hypersonic plasma flow field by application of an electromagnetic field. For the aspect of theoretical works, Khan et al. [17, 18] evaluate the exact solution of the unsteady flow of a generalized Brinkman type fluid under the effect of MHD in a channel. Moreover, they established a system of fractional order partial differential equations by choosing the Caputo-Fabrizio fractional derivative as a foundation to analysis MHD and nonlinear radiative heat flux.

However, till now, little information is available on the influences of electromagnetic flow control parameters to the MHD separation control effect. Moreover, the internal mechanisms lying in the MHD separation control system is not yet clear, and further researches are needed. This study is aimed at developing a comprehensive simulation tool for MHD hypersonic flow interaction. To discuss the effect of the electromagnetic field for hypersonic SWBLIs in laminar flows, this paper focuses on two configurations. Weakly ionized air flow control at hypersonic regimes have been simulated to estimate the potential effect of an MHD control system on separation flow configuration by application of an external electromagnetic field. The study provides a foundation for future developments of a comprehensive tool for hypersonic MHD separation flow control techniques.

\section{Governing Equations}

The coupling simulation of the electromagnetic field and the hypersonic plasma flow field can be accomplished by utilizing the compressible two-dimensional Navier-Stokes (N-S) equation with an electromagnetic source term [19] based on the low magnetic Reynolds number model, as seen in

$$
\frac{\partial \mathbf{U}}{\partial t}+\frac{\partial \mathbf{F}}{\partial x}+\frac{\partial \mathbf{G}}{\partial y}=\frac{\partial \mathbf{F}_{\mathrm{v}}}{\partial x}+\frac{\partial \mathbf{G}_{\mathrm{v}}}{\partial y}+\mathbf{S}_{\mathrm{MHD}},
$$

where $\mathbf{U}$ is the state vector of conservative variables. $\mathbf{F}, \mathbf{G}$ and $\mathbf{F}_{\mathrm{v}}, \mathbf{G}_{\mathrm{v}}$ are convective flux and viscous flux vectors in the $x, y$ coordinate directions, respectively. $\mathbf{S}_{\mathrm{MHD}}$ represents the electromagnetic source term vector, as expressed in Equation (7).

$$
\begin{aligned}
& \mathbf{U}=\left[\rho \rho u \rho v \rho e_{t}\right]^{T}, \\
& \mathbf{F}=\left[\begin{array}{llll}
\rho u & \rho u^{2}+p & \rho u v & \left(\rho e_{t}+p\right) u
\end{array}\right]^{T}, \\
& \mathbf{G}=\left[\begin{array}{llll}
\rho v & \rho v u & \rho v^{2}+p & \left(\rho e_{t}+p\right) v
\end{array}\right]^{T}, \\
& \mathbf{F}_{\mathrm{v}}=\left[\begin{array}{llll}
0 & \tau_{x x} & \tau_{x y} & u \tau_{x x}+v \tau_{x y}+q_{x}
\end{array}\right]^{T}, \\
& \mathbf{G}_{\mathrm{v}}=\left[\begin{array}{llll}
0 & \tau_{x y} & \tau_{y y} & u \tau_{x y}+v \tau_{y y}+q_{y}
\end{array}\right]^{T} \text {, } \\
& \mathbf{S}_{\mathrm{MHD}}=\left[\begin{array}{llll}
0 & (J \times B)_{x} & (J \times B)_{r} & J \cdot E
\end{array}\right]^{T},
\end{aligned}
$$

where

$$
\begin{aligned}
\rho e_{t} & =\rho \frac{\left(u^{2}+v^{2}\right)}{2}+\frac{p}{\gamma-1}, q_{n}=k_{L} \frac{\partial T}{\partial n}(n=x, y), \tau_{i j} \\
& =\mu_{L}\left(\frac{\partial u_{i}}{\partial x_{j}}+\frac{\partial u_{j}}{\partial x_{i}}\right)-\frac{2}{3} \mu_{L} \frac{\partial u_{k}}{\partial x_{k}} \delta_{i j} .
\end{aligned}
$$

The perfect gas thermal and viscous properties can be defined by the Sutherland formula:

$$
\begin{aligned}
& \mu_{L}(T)=1.4584 \times 10^{-6} \frac{(T)^{3 / 2}}{T+110.33}\left(\mathrm{~N} \cdot \mathrm{s} / \mathrm{m}^{2}\right), \\
& k_{L}(T)=2.4986 \times 10^{-3} \frac{(T)^{3 / 2}}{T+194.4}(\mathrm{~J} /(\mathrm{m} \cdot \mathrm{s} \cdot \mathrm{K})),
\end{aligned}
$$

where $\mu_{L}$ is the coefficient of viscosity and $k_{L}$ is the coefficient of thermal conductivity.

In computational space coordinate systems, Equation (1) can be expressed as

$$
\frac{\partial \widehat{\mathbf{U}}}{\partial \tau}+\frac{\partial \mathbf{F}}{\partial \xi}+\frac{\partial \widehat{\mathbf{G}}}{\partial \eta}=\frac{\partial \mathbf{E}_{\widehat{v}}}{\partial \xi}+\frac{\partial \widehat{\mathbf{G}}_{\mathrm{v}}}{\partial \eta}+\widehat{\mathbf{S}}_{\mathrm{MHD}},
$$

where

$$
\begin{aligned}
\widehat{\mathbf{U}} & =\bar{J} \cdot \mathbf{U}, \widehat{\mathbf{S}}_{\mathrm{MHD}}=\bar{J} \cdot \mathbf{S}_{\mathrm{MHD}}, \mathbf{E}=\bar{J} \cdot\left(\xi_{x} \mathbf{F}+\xi_{y} \mathbf{G}\right), \\
\widehat{\mathbf{G}} & =\bar{J} \cdot\left(\eta_{x} \mathbf{F}+\eta_{y} \mathbf{G}\right), \mathbf{F}_{\widehat{\mathrm{v}}}=\bar{J} \cdot\left(\xi_{x} \mathbf{F}_{\mathrm{v}}+\xi_{y} \mathbf{G}_{\mathrm{v}}\right), \\
\widehat{\mathbf{G}}_{\mathrm{v}} & =\bar{J} \cdot\left(\eta_{x} \mathbf{F}_{\mathrm{v}}+\eta_{y} \mathbf{G}_{\mathrm{v}}\right) .
\end{aligned}
$$

Here $\bar{J}$ is the Jacobian of transformation. The detail expressions of these terms can be found in Ref. [20]. Three 
additional variables are included in the above equations compared to the standard conservation equations without applying electromagnetic field, namely, magnetic induction strength $B$, electric strength $E$, and electric current density $J$ . The conservation of momentum equation and the total energy equation are modified with the inclusion of a Lorentz force $(J \times B)$ and Joule heating $(J \cdot E)$, respectively, which appear on the right side of the above equations. The current density can be evaluated from Ohm's law [21, 22].

$$
J=\sigma(E+\mathbf{U} \times B)
$$

It is assumed that the electrodes are ideal conductors; the external electrical field is given by Equation (13) and applied in $z$ direction.

$$
E=-k(\mathbf{U} \times B),
$$

where $k$ is the load factor, and $k>1$ for the MHD accelerator $[9,23]$.

Considering that the Hall effect is neglected, the electrical conductivity and external electromagnetic field are simplified to be constant in the MHD interaction zone. Except for the MHD zone, the electrical conductivity and electromagnetic field strength are assumed to be zero.

$$
\begin{gathered}
B_{x}=0, \quad B_{y}=B, \quad B_{z}=0, \\
E_{x}=0, \quad E_{y}=0, \quad E_{z}=-k B u, \\
J_{x}=0, \quad J_{y}=0, \quad J_{z}=\sigma(1-k) B u, \\
J \cdot E=J_{z} \cdot E_{z}=-\sigma k(1-k) u^{2} B^{2} .
\end{gathered}
$$

For the solution of Equation (10), the influence of the electromagnetic field is restricted exclusively to the electromagnetic source term. Thus, the solution procedure can take advantage of the ability to use more conventional computational fluid dynamics (CFD) methods. In this paper, the AUSMPW+ second-order upwind scheme with the MUSCL reconstruction method is used for the inviscid flux vectors. The diffusion terms are calculated by the second-order central-differencing scheme. As for time discretization, an approximately factored Lower-Upper Symmetric GaussSeidel (LU-SGS) scheme is used to eliminate the rigid problem caused by the large electromagnetic source terms.

\section{Code Validations and Grid Independency Test}

In order to validate the code's ability to accurately simulate the SWBLI in hypersonic laminar separation flows, a compression corner flow simulation was run for comparison to experimental results [24]. The freestream conditions were specified as initial conditions, as shown in Table 1. The incoming boundary layer is laminar and the Reynolds number at the corner is $10^{5}$; a cold wall $\left(T_{w}=297.2 \mathrm{~K}\right)$ is assumed, and a perfect-gas model for air is used. Figure 1 shows the grid diagrammatic sketch for ramp calculations, which consists of 101 grid points in the normal direction and 101 grid
TABLE 1: Freestream conditions for compression corner flow.

\begin{tabular}{lcc}
\hline Property & Symbol & Value \\
\hline Mach number & $\mathrm{Ma}$ & 14.1 \\
Temperature & $T_{\infty}$ & $72.7 \mathrm{~K}$ \\
Reynolds number & $\mathrm{Re} / \mathrm{m}$ & $2.32 \times 10^{5} / \mathrm{m}$ \\
Density & $\rho_{\infty}$ & $4.84 \times 10^{-4} \mathrm{~kg} / \mathrm{m}^{3}$ \\
\hline
\end{tabular}

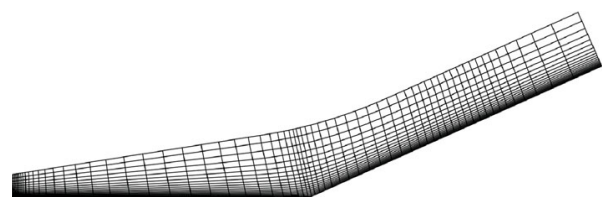

FIGURE 1: Grid diagrammatic sketch for ramp calculations.

points in the streamwise direction. Grid clustering has been implemented near the corner and the wall region to accurately capture viscous effects.

In Figure 2(a), the wall pressure and skin-friction distributions for high-Mach-number flows over a 15-degree ramp are plotted. Figure 2(b) shows the comparison of the wall pressure and skin-friction distributions with experimental values over an 18-degree ramp. Very good agreement was found for the cases of a 15-degree ramp and an 18-degree ramp. Here, the pressure coefficient is defined as $C_{p}=[p /(1 /$ 2) $\left.\rho_{\infty} u_{\infty}^{2}\right]$ and the skin-friction coefficient is defined as $C_{f}=$ $\left[\tau_{w} /(1 / 2) \rho_{\infty} u_{\infty}^{2}\right]$.

Figure 3 shows the computed pressure and typical heat transfer distributions for a 24-degree ramp case. Compared with experimental results provided by Holden et al., there are only very slight differences in the predictions from the codes. The general features of the computed results for the 24-degree ramp case demonstrated a trend that the extent of separation is slightly larger than that found in the experiment. The surface heat transfer is represented in a nondimensional form by the Stanton number defined as

$$
\mathrm{St}=\frac{\dot{q}_{w}}{\rho_{\infty} U_{\infty}\left(h_{s t, \infty}-h_{w}\right)},
$$

where $\rho_{\infty}, U_{\infty}$ are the density and velocity of the upstream flow, respectively; $\dot{q}_{w}$ is the wall heat transfer (in $\left.\mathrm{W} / \mathrm{m}^{2}\right) ; h_{s t, \infty}$ is the upstream flow stagnation enthalpy; and $h_{w}$ is the flow enthalpy at the wall. Nevertheless, although the location of the peaks of the surface pressure is not the same, the overall results match up well.

In order to guarantee the accuracy of the solver's Lorentz source term, a flat plate flow simulation was run for comparison to reference results. The flow parameters for this test case are shown in Table 2. The magnetic Reynolds number based on the length of the flat plate is $\mathrm{Re}_{m}=0.058$. Therefore, the MHD equations will be solved by the low magnetic Reynolds number formulation. A structural grid consisting of 50 grid points in the normal direction and 100 grid points in the streamwise direction was used to compute the case; the distance of the first grid point off the wall is $2 \times 10^{-7} \mathrm{~m}$. 


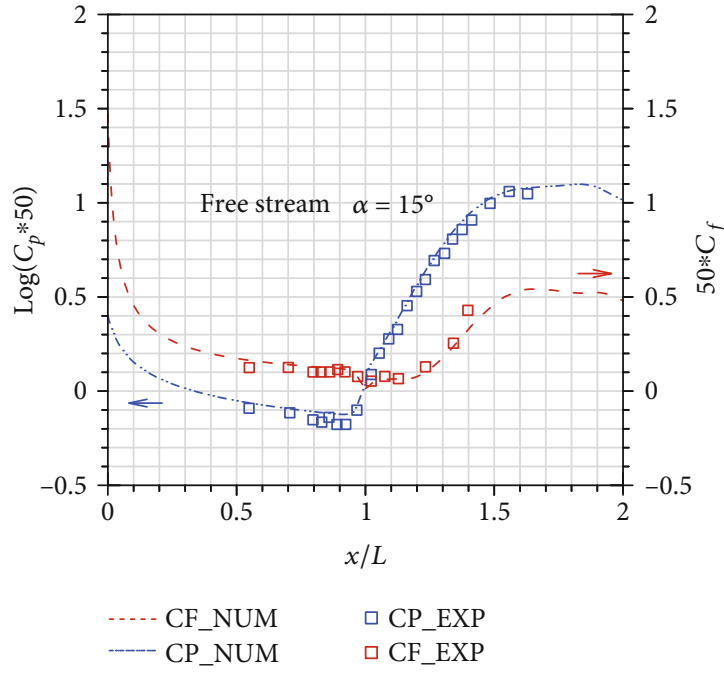

(a)

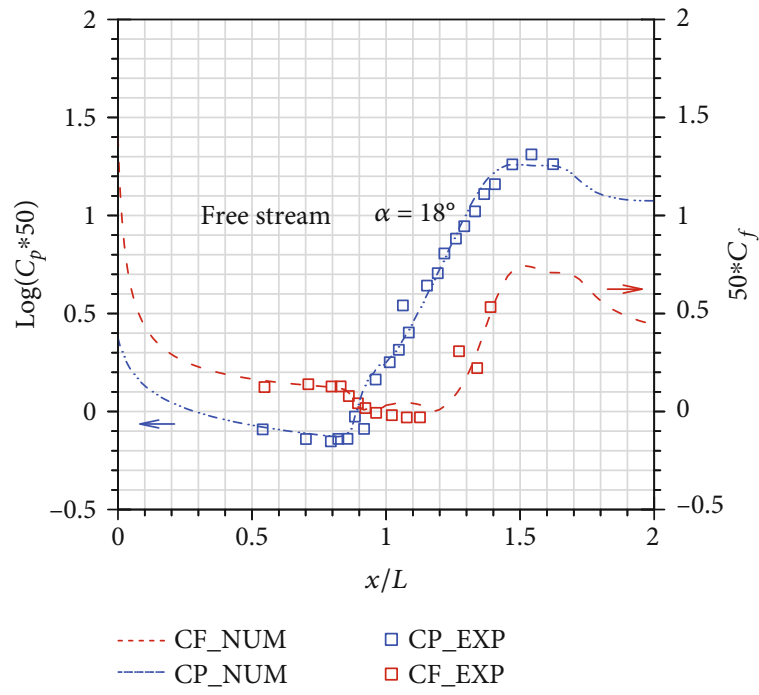

(b)

FIGURE 2: Comparison between pressure and skin friction for compression corner flows and experimental data provided by Holden: (a) ramp angle $15^{\circ}$; (b) ramp angle $18^{\circ}$.

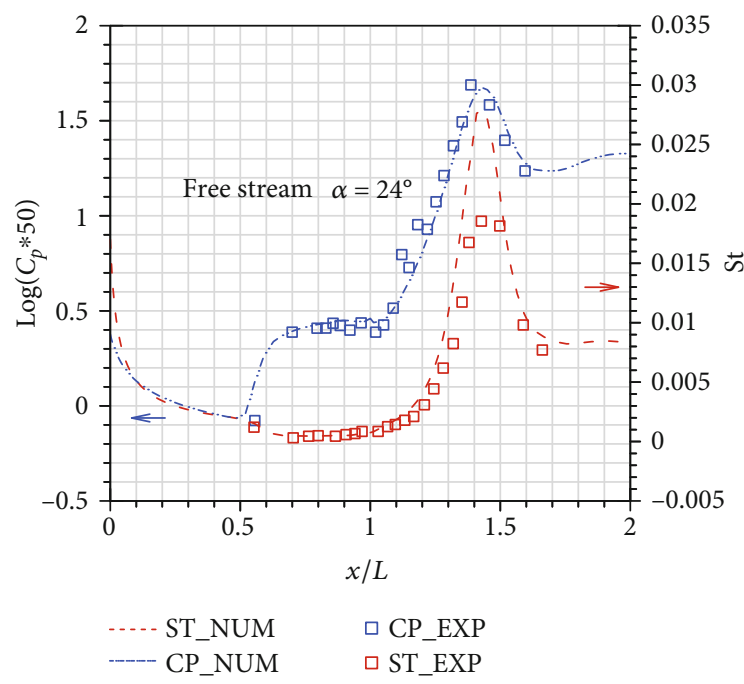

Figure 3: Comparison between pressure and heat transfer in $24^{\circ}$ wedge-induced separated flows and experimental data provided by Holden.

TABLE 2: Freestream conditions for flat plate flow.

\begin{tabular}{lcc}
\hline Property & Symbol & Value \\
\hline Mach number & $\mathrm{Ma}$ & 2.0 \\
Temperature & $T_{\infty}$ & $300 \mathrm{~K}$ \\
Reynolds number & $\operatorname{Re}_{\infty}$ & $3.75 \times 10^{6}$ \\
Pressure & $P_{\infty}$ & $1.706 \times 10^{5} \mathrm{~N} / \mathrm{m}^{2}$ \\
Length of flat plate & $L$ & $0.08 \mathrm{~m}$ \\
Electrical conductivity & $\sigma_{e}$ & $800 \mathrm{~S} / \mathrm{m}$ \\
\hline
\end{tabular}

The velocity at $x=0.06 \mathrm{~m}$ under the applied magnetic field strength of $1.0 \mathrm{~T}$ is illustrated in Figure 4 . It shows that the velocity profiles obtained in this paper are in good agreement with Hiromasa's simulation results [25] and thus validates the MHD code's ability of numerical simulation in the low magnetic Reynolds number flow condition.

It is widely considered that a major source of inaccuracy in aerodynamic prediction is associated with grid scale, especially the normal grid spacing at the wall. The authors, therefore, validated the grid independency in advance. The blunt body Orbital Reentry Experiment (OREX) capsule is utilized as the calculation model. Figure 5 shows the configuration of OREX. The forebody shape is composed of a spherical nose, a cone, and a circular shoulder. A grid independency test is conducted by employing six grids with different normal grid spacings at the wall when $\mathbf{B}_{0}$ equals $0.0 \mathrm{~T}$. For this case, the flight conditions correspond to the altitude of $51.99 \mathrm{~km}$ and a velocity of $3873.4 \mathrm{~m} / \mathrm{s}$. Heat flux distributions under different normal grid spacings at the wall are shown in Figure 6; it is clear that the grid is convergent for heat flux computations while inflow cell Reynolds number $\operatorname{Re}_{\Delta n, \infty}=1.6$, and a proper grid is employed in the following study.

\section{Ramp-Induced Separation Control}

When a hypersonic flow propagates over a compression ramp, a remarkable adverse pressure gradient may appear near the corner due to the displacement effect of the wall, possibly leading to a separation in the vicinity of the corner, as well as the emergence of the shock wave boundary layer interaction. The separation could result in a variety of unfavorable factors in engineering applications. An effective tool to reduce flow separation is to introduce a Lorentz force on the wall near the corner. Table 3 shows the parameters of MHD flow over the $24^{\circ}$ compression corner. A structural grid is generated for this compression ramp case, which consists 


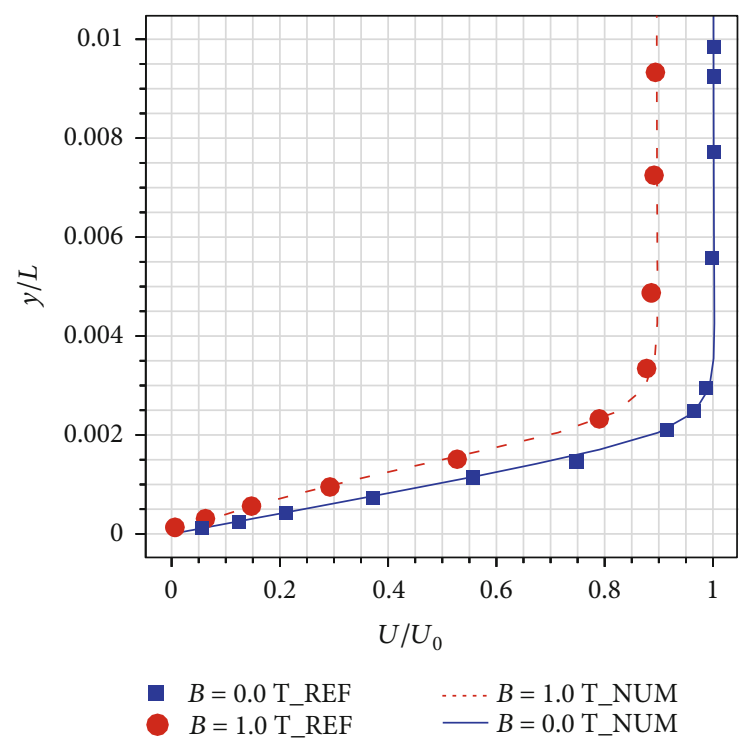

FIGURE 4: Laminar velocity profiles at $x=0.06 \mathrm{~m}$.

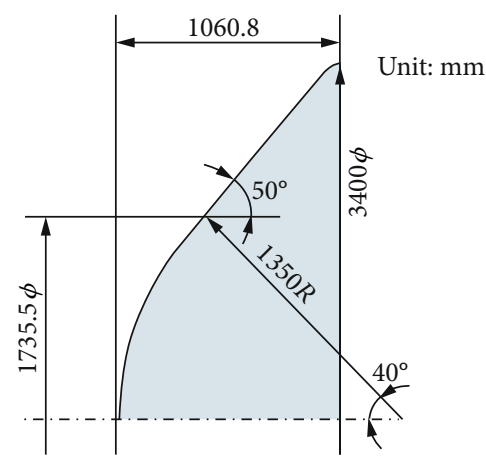

FIgURE 5: Geometry for the OREX.

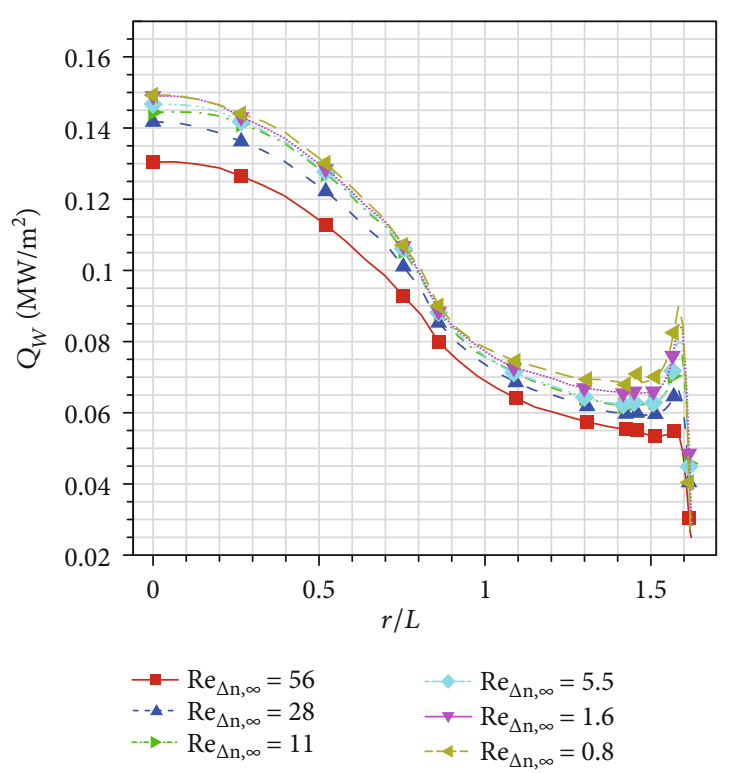

Figure 6: Heat flux distributions under different normal grid spacing at the wall.
TABLE 3: Parameters of MHD flow over the $24^{\circ}$ compression corner.

\begin{tabular}{lcc}
\hline Property & Symbol & Value \\
\hline Mach number & $\mathrm{Ma}$ & 7.7 \\
Temperature & $T_{\infty}$ & $300 \mathrm{~K}$ \\
Reynolds number & $\mathrm{Re} / \mathrm{m}$ & $7.60 \times 10^{4} / \mathrm{m}$ \\
Density & $\rho_{\infty}$ & $5.27 \times 10^{-4} \mathrm{~kg} / \mathrm{m}^{3}$ \\
\hline
\end{tabular}

of 101 grid points in the normal direction and 101 grid points in the streamwise direction. The grid points are clustered near the wall and the distance of the first grid point off the wall is $1 \times 10^{-6} \mathrm{~m}$. In this section, these issues are illustrated with relatively simple two-dimensional interaction flows. The simulations are used to describe the key features of hypersonic SWBLI flows. These simulations may not capture all of the critical physics of the flows. However, the simulations are useful for understanding many important aspects of these complex flows.

Figure 7 illustrates the schematic of electric field and magnetic field in the MHD interaction zone. A suitable configuration of magnets and electrodes can generate a body force within an electrically conducting fluid that influences the boundary layer. Figure 8 shows the schematic sketch of the MHD separation flow control system that allows the generation of a streamwise Lorentz force, where $\mathbf{B}$ represents the magnetic field vector, directed parallel to $y$-axis; $\mathbf{U}$ is the flow velocity vector; and $\mathbf{J}$ represents the current density vector in the region of MHD interaction. The Lorentz force vector is defined by the relation $\mathbf{F}=\mathbf{J} \times \mathbf{B}$, which points to the streamwise direction.

The effect of the MHD interaction region location and the applied electromagnetic field strength are investigated in this paper. The electromagnetic flow control parameters on SWBLI are summarized in Table 4. Three kinds of electromagnetic configurations are compared and analyzed. The electromagnetic configuration MHD1 is where an electromagnetic field is applied over a region near the separation point from $x_{1} / L=0.5$ to $x_{2} / L=0.8$ on the flat plate section. Another area used is a zone adjacent to the corner from $x_{1}$ / $L=0.9$ to $x_{2} / L=1.2$, namely, the electromagnetic configuration MHD2. As to the electromagnetic configuration MHD3, the MHD control system was implemented in a zone that is near the reattachment point from $x_{1} / L=1.0$ to $x_{2} / L=1.3$.

The goal of applying an electromagnetic field into the flow is to control the flow separation that occurs due to a sufficiently highly adverse pressure gradient and a loss of the momentum by the friction. The case of separation induced by a compression ramp is illustrated by the flow sketch in Figure 9. The compression corner causes the boundary layer to separate, producing a separation shock, reattachment shock, and separation zone. It can also be seen from Figure 9 that the position of the separation point $\mathbf{S}$ is near $x$ $/ L=0.67$ and that of the reattachment point $\mathbf{R}$ is near $x / L$ $=1.25$. For MHD analysis, Figure 10 illustrates the flow field structure induced by a ramp with a localized MHD flow control system MHD2. The streamwise Lorentz force generated after the application of electromagnetic field has resulted in 


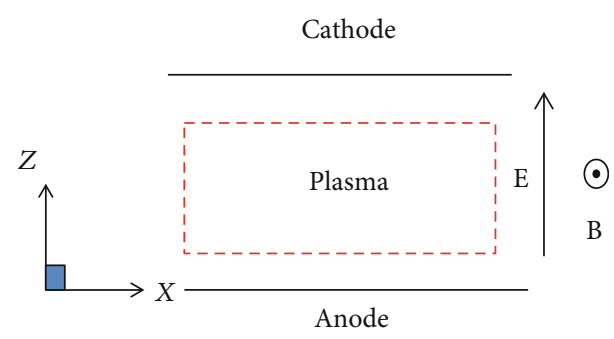

Figure 7: Schematic of electric field and magnetic field in MHD interaction zone.

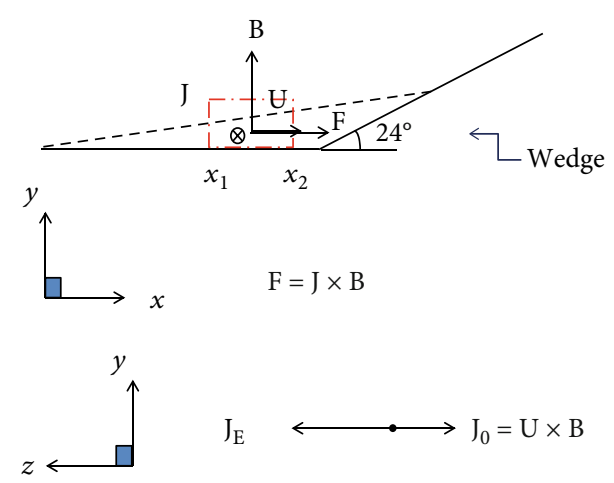

FIGURE 8: Schematic sketch of MHD separation flow control over a 24-degree ramp.

TABLE 4: Electromagnetic flow control parameters on SWBLI.

\begin{tabular}{lccccc}
\hline Case & $x_{1} / L$ & $x_{2} / L$ & $B_{\max }(\mathrm{T})$ & Load factor & Sigma \\
\hline MHD1 & 0.5 & 0.8 & 2.5 & 1.5 & 2.0 \\
MHD2 & 0.9 & 1.2 & 2.5 & 1.5 & 2.0 \\
MHD3 & 1.0 & 1.3 & 2.5 & 1.5 & 2.0 \\
\hline
\end{tabular}

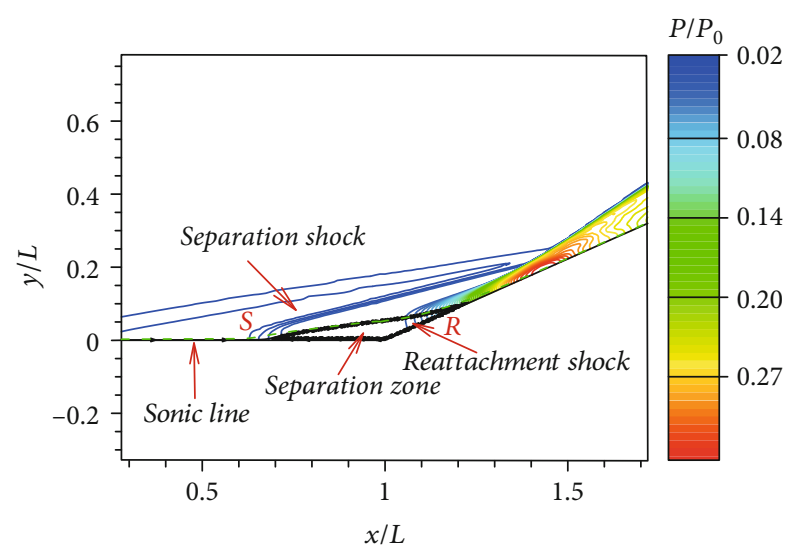

Figure 9: Flow field structure induced by a ramp without MHD control.

a decrease in the size of the separation zone. With MHD accelerating the boundary layer, the separation in the compression corner is eliminated.

In order to distinguish the effects of Joule heating from the effects of the Lorentz force, the flow field electrical conductivity is brought down from $2.0 \mathrm{mho} / \mathrm{m}$, in the previous

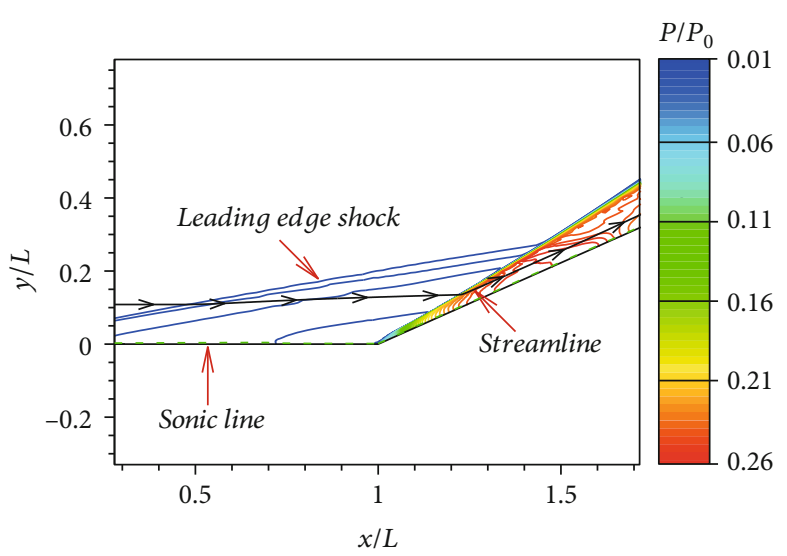

Figure 10: Flow field structure induced by a ramp with MHD control (MHD2).

case (Table 4), to $1.0 \mathrm{mho} / \mathrm{m}$. In this case, we imposed an electric field of load factor $k=1.5$ and magnetic field of $2.5 \mathrm{~T}$ in the MHD interaction zone. The effects of the Joule heating on the wall pressure and wall heat transfer distributions are presented in Figure 11. The simulated wallpressure distribution for both the Joule heating term and the Lorentz force was found almost the same as the one for no MHD interaction under the freestream conditions of Table 3. This is explained by the increase of wall pressure by Joule heating and by the decrease of wall pressure due to flow acceleration by the streamwise Lorentz force, which agrees well with findings in Ref. [26]. The effects of the accelerating Lorentz force are not strong enough to nullify the negative effects of Joule heating. For the case without the Joule heating term, the significant pressure decrease and wall heat transfer increase were observed.

The influences of the MHD interaction region location on the wall pressure distributions are summarized in Figure 12(a), including without MHD interaction case. In the case with MHD off, the wall pressure distribution initially exhibits a steep rise, associated with separation, followed by a plateau typical of separated flows. After that, a more progressive pressure rise occurs during reattachment. For the viscous MHD computation, case MHD1 suppresses the plateau typical of wall-pressure distribution locally, where the electromagnetic field is applied. Moreover, the plateau pressure is increased, and the location of the wall pressure peak changed. Case MHD2 manages to completely eliminate the plateau typical of wall-pressure distribution. As to case MHD3, the applied electromagnetic field moves downstream of the location of separation point $\mathbf{S}$ and moves upstream of the location of reattachment point $\mathbf{R}$.

Figure 12(b) depicts the influence of the MHD interaction region location on the wall heat transfer distribution for ramp-induced separation flow with and without the MHD control effect. When the MHD is off, a rapid decrease starts at a location coincident with the separation onset. This decrease is typical of ramp-induced separation in laminar flows. The heat transfer experiences a minimum in the separated region, then rises during reattachment; the peak value is achieved downstream of the reattachment point. For the 


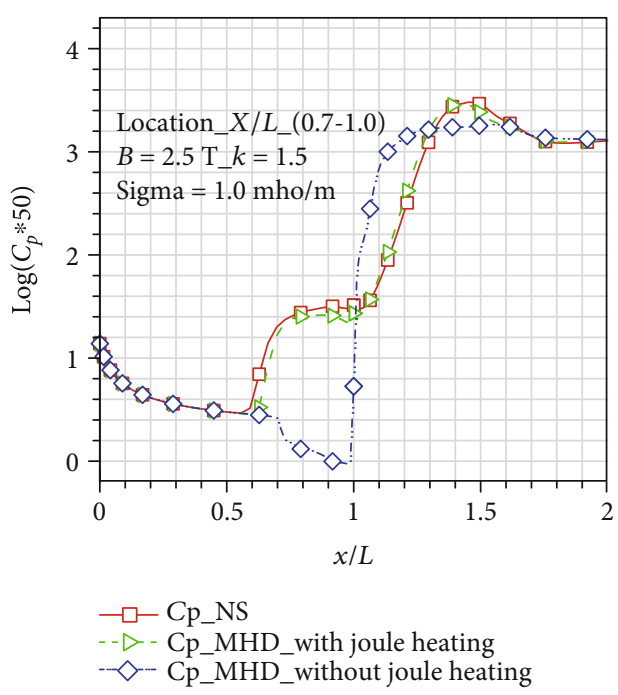

(a)

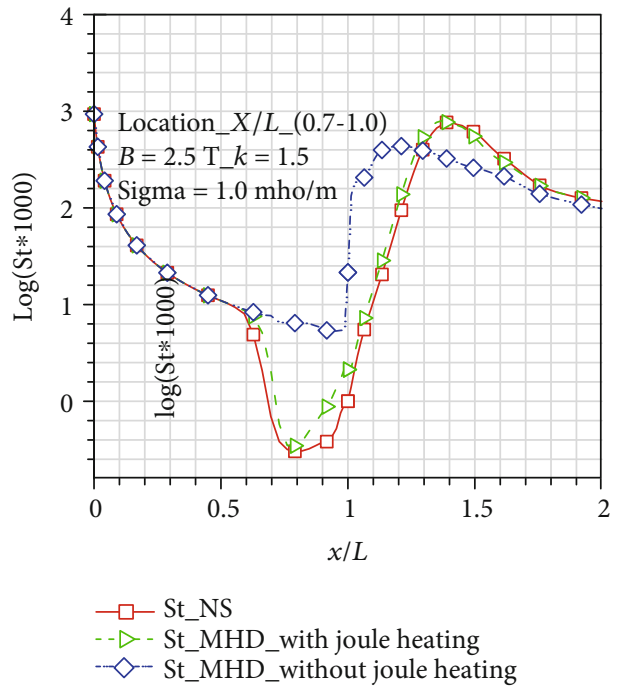

(b)

FIGURE 11: Effect of the Joule heating on the wall pressure and wall heat transfer distribution: (a) wall pressure distribution; (b) wall heat transfer distribution.

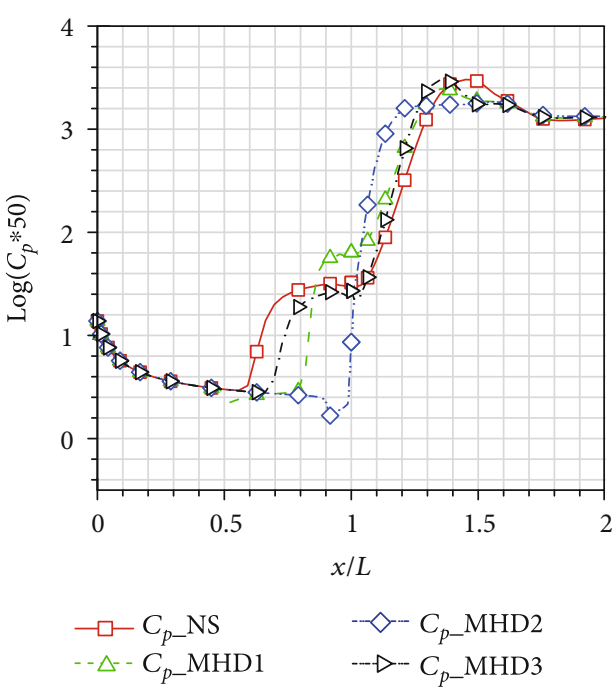

(a)

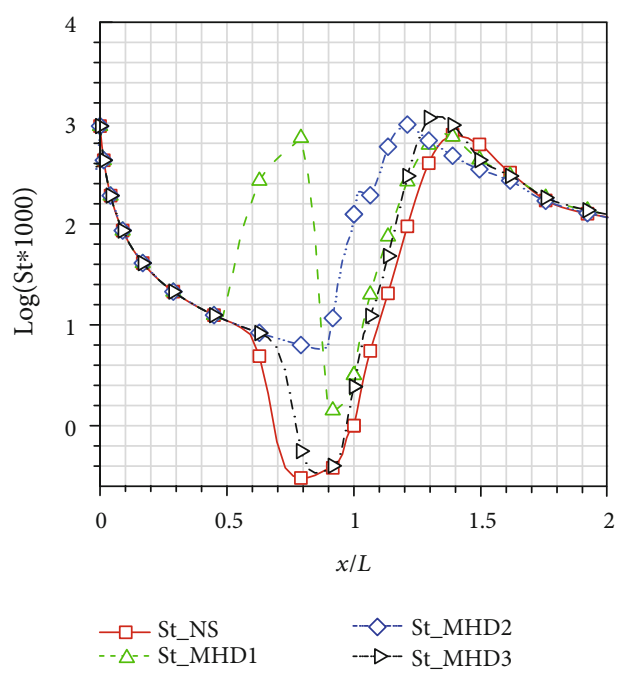

(b)

FIGURE 12: Effect of the interaction region location on the wall pressure and wall heat transfer distribution: (a) wall pressure distribution; (b) wall heat transfer distribution.

viscous MHD computation, the acceleration of the flow near the separation point causes a spike in the wall heat transfer for case MHD1. All in all, there exists a best location for the MHD zone to be applied and completely eliminate the separation of the flow from the surface (MHD2).

The velocity and temperature profiles are plotted at $x / L$ $=0.9$, shown in Figure 13. In this picture, the simulation results for different electromagnetic field locations are represented by various lines in the profile plot. The computation showed that the boundary layer is accelerated to more full compare to the case with MHD off, but the velocity and temperature profiles vary greatly with different MHD interaction region locations. The velocity profiles are most full when the MHD interaction region location is at $0.9<x / L<1.2$
(MHD2). It can be found that the boundary-layer thickness decreased when the MHD is applied.

In our simulations, we consider several combinations of electric and magnetic fields to understand the effects of these external fields on the boundary layer structure. The effects of the electromagnetic field strength on the wall pressure distributions are shown in Figure 14(a). In the plot, the electric field intensity is represented by the load factor $k$. The Joule heating and Lorentz force become greater and alter the flow to a greater extent than with the low electromagnetic field intensity. It can be seen that the pressure plateau grows smaller with strengthening of the electromagnetic field strength, and the flow field structure can be changed. Moreover, the plateau typical of wall-pressure distribution can be 


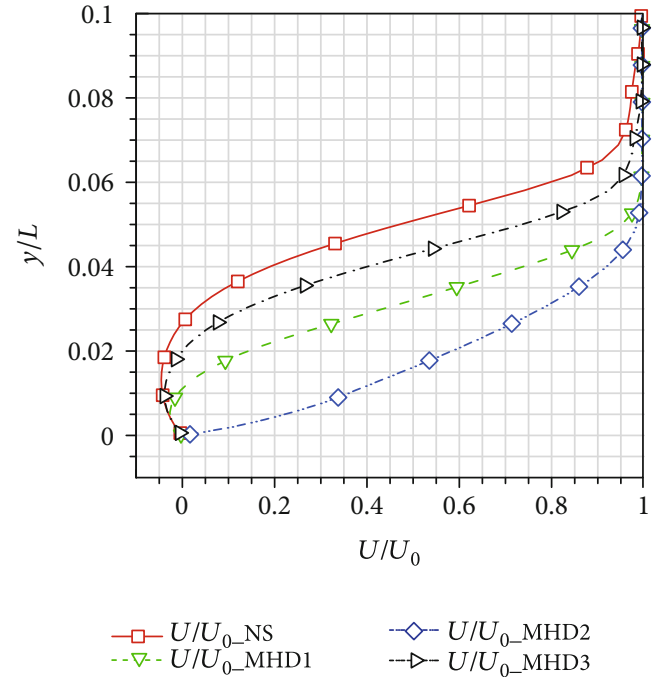

(a)

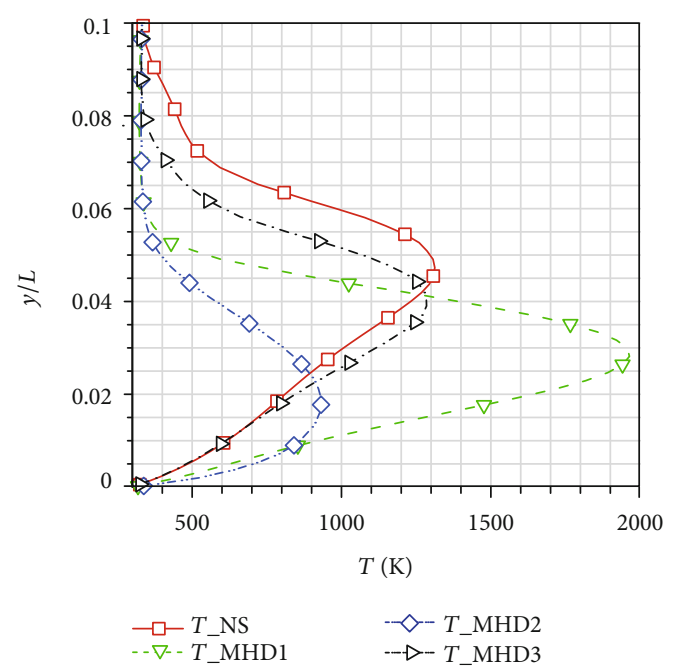

(b)

FIGURE 13: Velocity and temperature profiles near the corner at $x / L=0.9$ under different interaction region locations: (a) profiles of velocities; (b) profiles of temperatures.

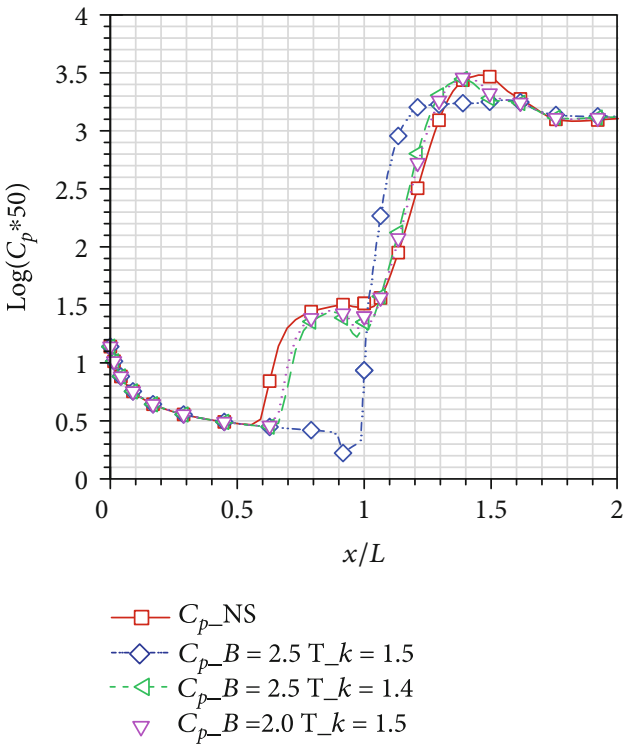

(a)

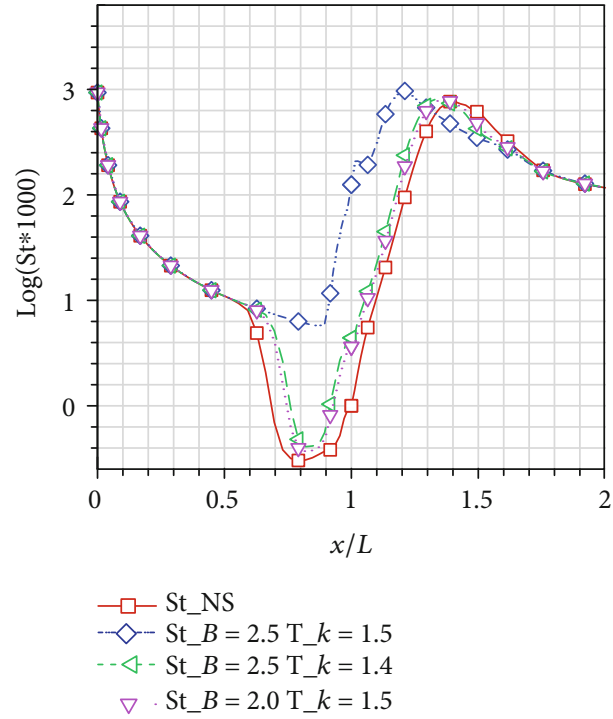

(b)

FIGURE 14: Effect of the electromagnetic field strength on the wall pressure and wall heat transfer distribution: (a) wall pressure distribution; (b) wall heat transfer distribution.

completely eliminated with the magnetic field strength of $2.5 \mathrm{~T}$ and the load factor of 1.5. The effects of the electromagnetic field strength on the wall heat transfer distributions are summarized in Figure 14(b). As we can see, the computational case with the magnetic field strength of $2.5 \mathrm{~T}$ and the load factor of 1.5 shows higher heat transfer values near the corner. All other computational cases does not provide a substantial reduction in the wall heat transfer peak but move the location of the wall heat transfer peak.

The velocity and temperature profiles near the corner at $x / L=0.9$ under different electromagnetic field strengths are shown in Figure 15. It can be seen that the velocity and temperature profiles vary drastically with different electromagnetic field strengths. The velocity profiles are most full when the magnetic field strength $B=2.5 \mathrm{~T}$ and load factor $k=1.5$. Moreover, the application of these combinations of electric and magnetic field strengths is able to accomplish the goal of the present work to eliminate the ramp-induced separation. For mitigating the effects of Joule heating, the electromagnetic field strength required is significant, and it is observed that the effects of the accelerating Lorentz force are strong enough to nullify the effects of Joule heating. 


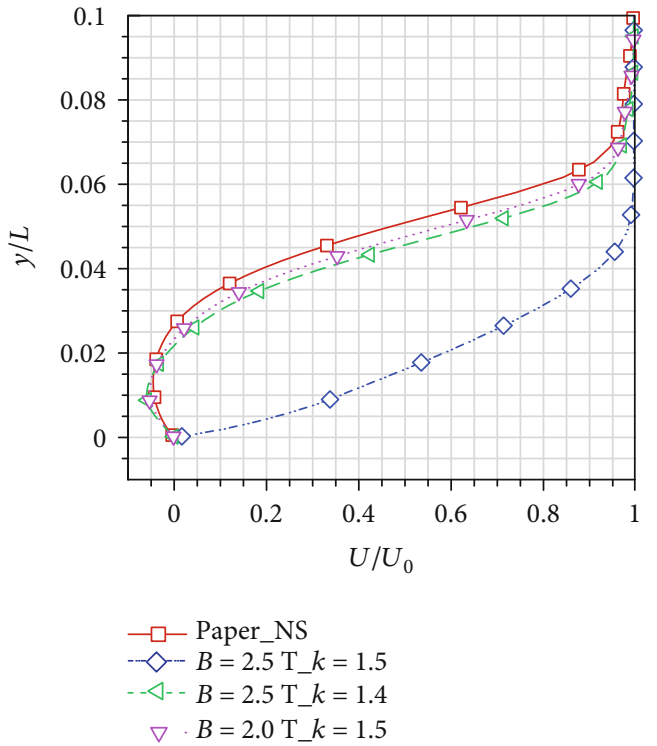

(a)

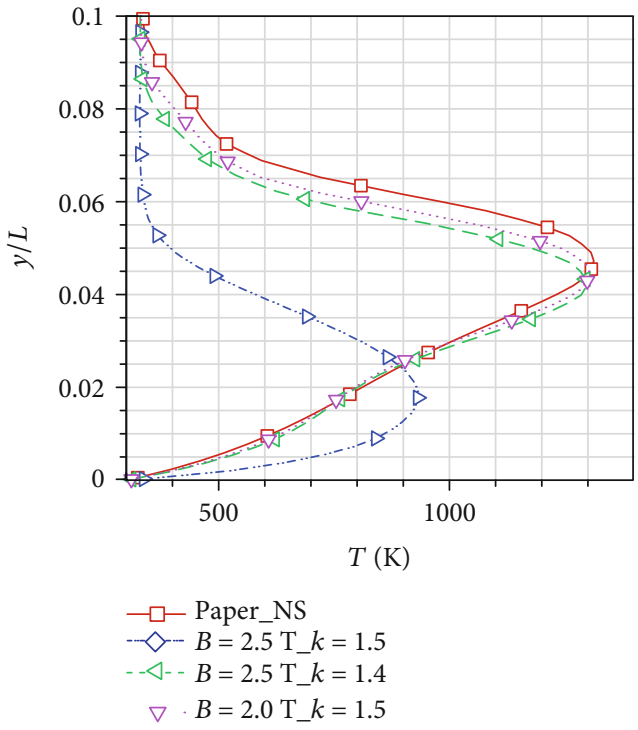

(b)

Figure 15: Velocity and temperature profiles near the corner at $x / L=0.9$ under different electromagnetic field strengths: (a) profiles of velocities; (b) profiles of temperatures.

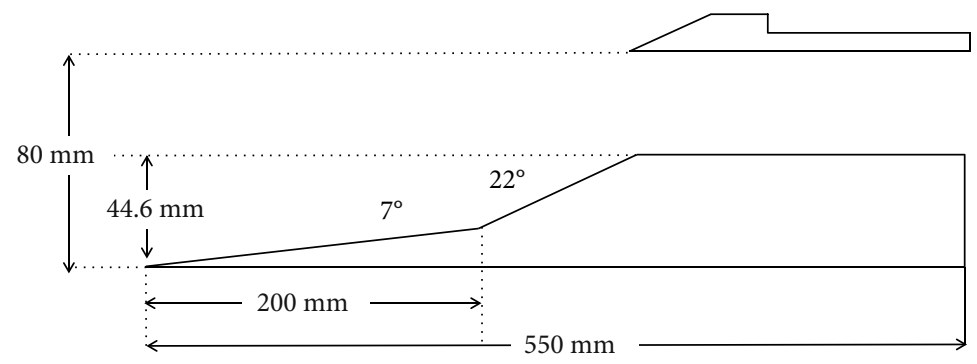

FIGURE 16: Geometry for the 2D double wedge ramp scramjet inlet (mm).

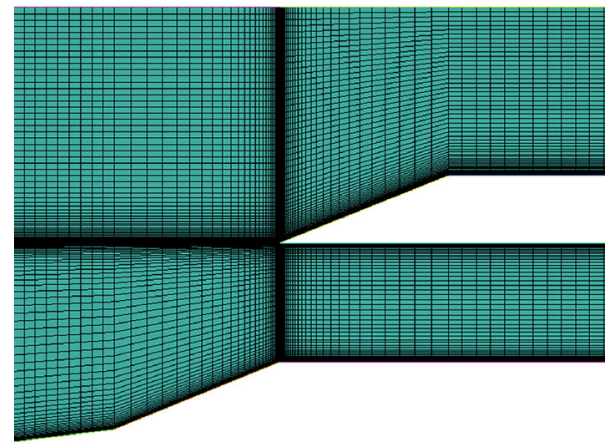

FIGURE 17: Local grid for scramjet inlet calculations.

TABle 5: Flow conditions at the scramjet inlet entrance.

\begin{tabular}{lcc}
\hline Property & Symbol & Value \\
\hline Mach number & $\mathrm{Ma}$ & 5.8 \\
Temperature & $T_{\infty}$ & $300 \mathrm{~K}$ \\
Reynolds number & $\mathrm{Re} / \mathrm{m}$ & $5.75 \times 10^{4} / \mathrm{m}$ \\
Density & $\rho_{\infty}$ & $5.27 \times 10^{-4} \mathrm{~kg} / \mathrm{m}^{3}$ \\
\hline
\end{tabular}

\section{Inlet Boundary Layer Separation Control}

For a $2 \mathrm{D}$ double wedge ramp hypersonic inlet, the reflect shock impinges on the shoulder and interacts with the thick boundary layer growing along the ramp side wall, producing a classic shock-induced separation phenomenon. The mechanisms of boundary layer separation caused by SWBLI in the scramjet inlet and its control with magnetohydrodynamics were investigated numerically with a two-dimensional CFD code. Figure 16 presents the geometry for the 2D double wedge ramp scramjet inlet. The local grid for scramjet inlet calculations is shown in Figure 17. The 2D double wedge ramp scramjet inlet structural grid is divided into eight blocks, which consist of 43200 grid points. Grid clustering has been implemented near the wall region, and the distance of the first grid point off the wall is $1 \times 10^{-6} \mathrm{~m}$.

For numerical simulation, freestream conditions at the scramjet inlet entrance are shown in Table 5. In the experimental case of low Reynolds number and low turbulence intensity, the boundary layer downstream of the inlet lip can be assumed as a laminar boundary layer. In Section 4, it has been verified that the separation flow control system can alleviate or even completely eliminate the ramp- 


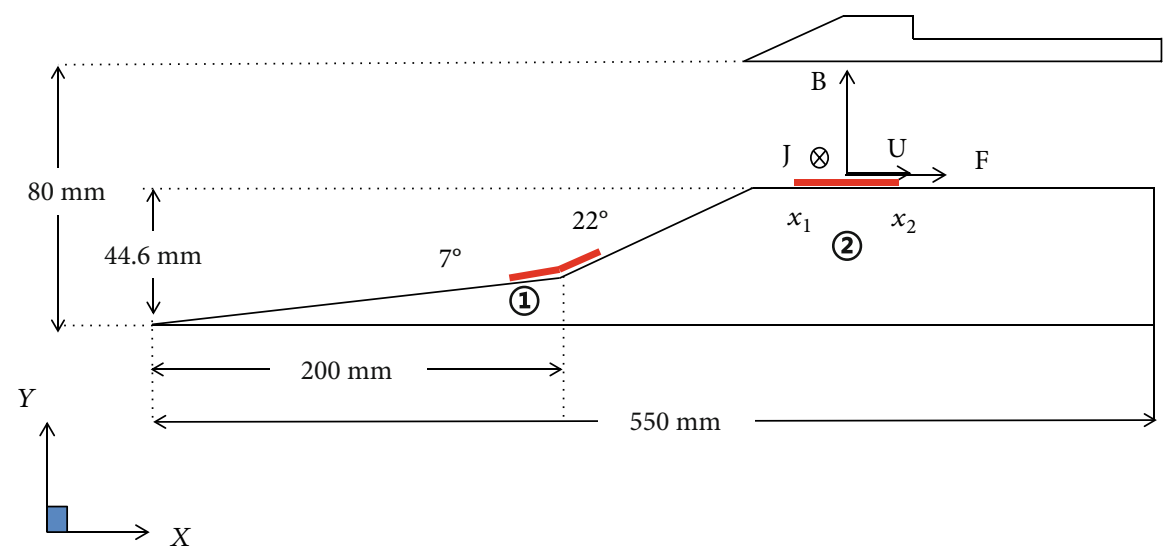

FIGURE 18: Schematic sketch of MHD separation flow control over a hypersonic inlet.

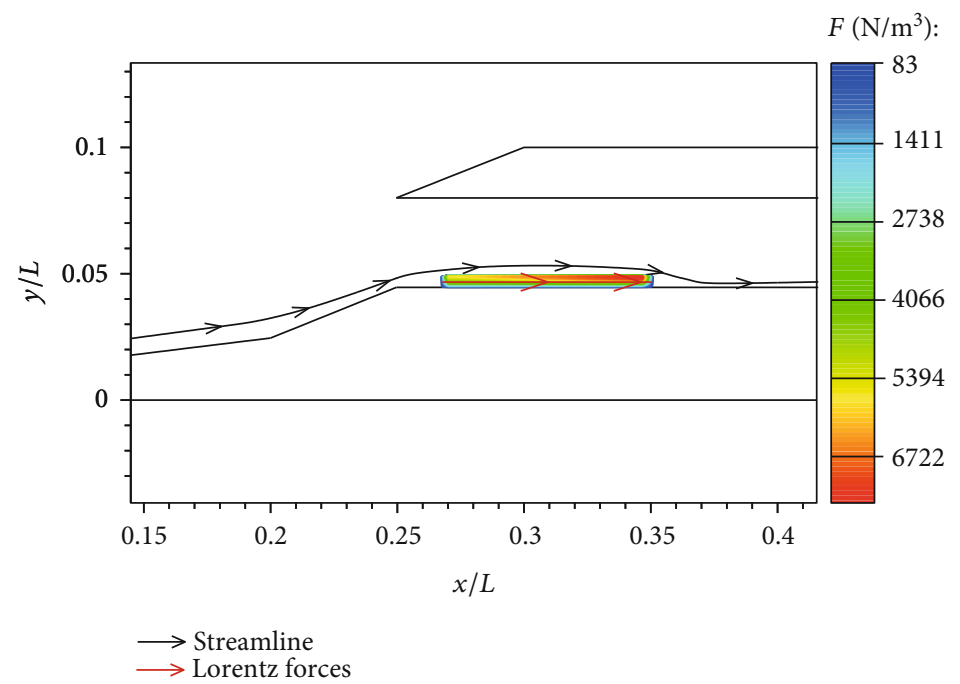

FIGURE 19: Local Lorentz force and streamline distributions of MHD separation flow control system $(B=3.0 \mathrm{~T})$.

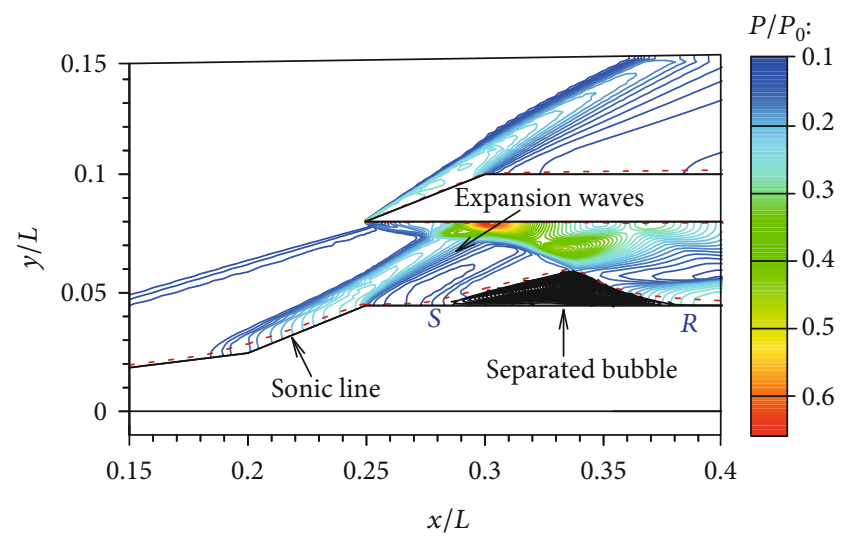

FIGURE 20: Flow field structure in hypersonic inlet without MHD control.

induced separation, as shown in Figure 18 control system (1). To extend the local laminar region and eliminate the shockinduced separation bubble, a localized MHD flow control system (2) should be applied to accelerate the boundary layer in the 2D double wedge ramp hypersonic inlet. Figure 19 dis-

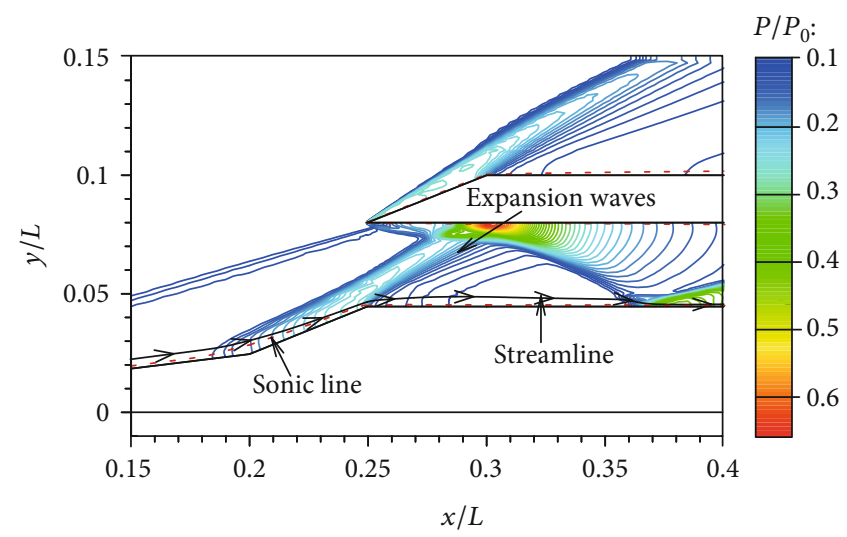

FIgURE 21: Flow field structure in hypersonic inlet with MHD control $\left(B_{\max }=3.0 \mathrm{~T}\right)$.

plays the Lorentz force and streamline distributions of MHD separation flow control system (2). In this case, we have imposed an external uniform magnetic field strength of $3.0 \mathrm{~T}$, directed parallel to the $y$-axis in the MHD interaction control region. It can be seen that the MHD separation flow 


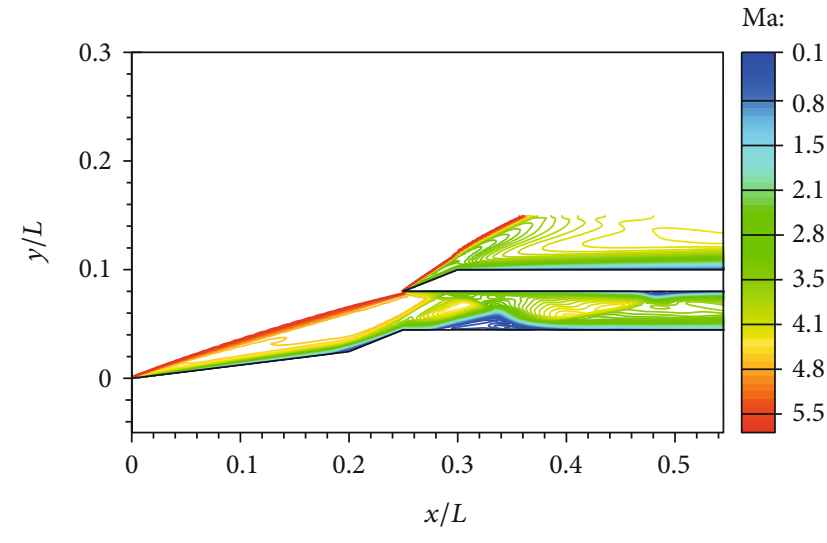

(a)

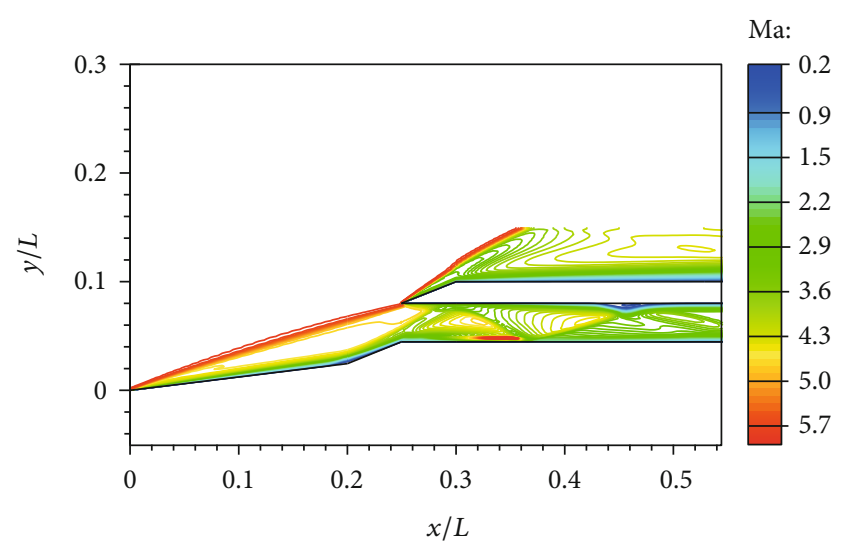

(b)

FIGURE 22: Mach contours in a 2D double wedge ramp hypersonic inlet: (a) flow field without MHD control; (b) flow field with MHD control.

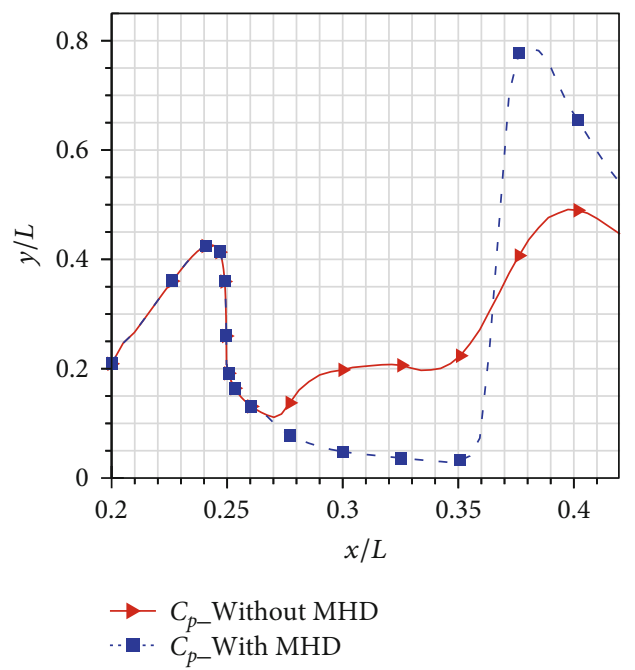

FIGURE 23: Wall pressure distribution in a 2D double wedge ramp hypersonic inlet.

control system (2) is mainly determined by the influence of the streamwise Lorentz force. The streamwise Lorentz force accelerates the flow and decreases the adverse pressure gradient, whereby the shock-induced separation control condition shall be achieved.

Figure 20 is a computational simulation of a $2 \mathrm{D}$ double wedge ramp hypersonic inlet configuration without the MHD control. The figure shows a classic SWBLI, with the impinging shock reflecting from the surface. The shock impingement causes the boundary layer to separate and produce a separation bubble. $\mathbf{S}$ and $\mathbf{R}$ represent the separation point and reattachment point, respectively. The recirculating flow separates from the freestream flow bounded by a sonic line. Figure 21 illustrates the flow field structure in hypersonic inlet with MHD control system (2). As we can see, the MHD control technique could move the separation point downstream, diminish the separation bubble, and then alleviate or even eliminate the shock-induced separation.

Furthermore, a comparison of Mach contours has also been made for the computations without and with the appli- cation of electromagnetic field. From Figure 22, we can clearly observe that with an external electromagnetic field applied, the low velocity fluid in the boundary layer can be accelerated. The significant increase of the Mach number in the boundary layer was observed when the MHD control technique was applied.

Figure 23 plots the computed pressure distribution on the surface for hypersonic inlet case with and without MHD control effect. Here, the plateau typical of wall pressure is caused by the additional flow-turning due to the boundary layer and the complex system of shock waves generated by the interaction at the location from $x / L=0.25$ to $x / L=0.35$. Then, the MHD controlled case demonstrates that the shock-induced flow separation can be effectively reduced by flow acceleration of the Lorentz force directed in the streamwise direction. The shock-induced plateau typical of wall-pressure distribution can be completely eliminated.

\section{Conclusions}

SWBLI can be viewed as a competition between a variable property flow and an abrupt pressure rise. The result of this conflict depends on the pressure-rise amplitude and the boundary-layer characteristics. The possibility of controlling the hypersonic separation flows by application of an external electromagnetic field is investigated in this paper. Conclusions can be summarized as follows:

(1) The performance of MHD separation flow control is mainly determined by flow acceleration of the Lorentz force directed in the streamwise direction. The Joule heating term always brings negative effects on the MHD separation flow control and increases the static pressure locally, where the electromagnetic field is applied

(2) With an external electromagnetic field applied, the low velocity fluid in the boundary layer can be accelerated

(3) The MHD control technique could move the separation point downstream, diminish the separation 
bubble, and then alleviate or even eliminate the ramp-induced/shock-induced separation

(4) There exists a best location for the magnetohydrodynamic zone to be applied and completely eliminate the separation of the flow from the surface

The numerical simulation demonstrated that it is possible to suppress the separation bubble induced by shock wave boundary layer interactions with MHD accelerating the boundary layer. The study provides a foundation for future developments of a comprehensive tool for a hypersonic inlet MHD flow control technique.

\section{Data Availability}

The data that support the findings of this study are available within the article.

\section{Conflicts of Interest}

The authors declare that they have no conflicts of interest.

\section{Acknowledgments}

This work was supported by the National Key Research and Development Plan of China (No. 2019YFA0405203 and No. 2019YFA0405300).

\section{References}

[1] H. Babinsky and J. K. Harvey, Shock Wave Boundary Layer Interactions, Cambridge University Press, New York, 2011.

[2] W. Z. Xie, Z. M. Wu, A. Y. Yu, and S. Guo, "Control of severe shock-wave/boundary-layer interactions in hypersonic inlets," Journal of Propulsion and Power, vol. 34, no. 3, pp. 614-623, 2018.

[3] D. M. Van Wie and A. Nedungadi, "Plasma aerodynamic flow control for hypersonic inlets," in 40th AIAA/ASME/SAE/ASEE Joint Propulsion Conference and Exhibit, pp. 11-14, July, Fort Lauderdale, Florida, 2004.

[4] S. Zhang, G. Hong, D. Y. Zhang, and F. Q. Cheng, "Progress in hypersonic inlet flow controls by magnetohydrodynamic," Applied Mechanics and Materials, vol. 730, pp. 311-315, 2015.

[5] F. Falempin, A. A. Firsov, D. A. Yarantsev, M. A. Goldfeld, K. Timofeev, and S. B. Leonov, "Plasma control of shock wave configuration in off-design mode of $\mathrm{M}=2$ inlet," Experiments in Fluids, vol. 56, no. 3, article 54, 2015.

[6] Y. W. Li, B. L. Zhang, Y. H. Li, L. H. Xiao, Y. T. Wang, and G. Q. He, "Review on the application and prospect of magnetohydrodynamics in aeronautical engineering," Advances in mechanics, vol. 47, pp. 452-502, 2017.

[7] T. Fujino and Y. Shimosawa, "Numerical study of magnetohydrodynamic flow control along superorbital reentry trajectories," Journal of Spacecraft and Rockets, vol. 53, pp. 528-537, 2016.

[8] J. F. Dietiker and K. A. Hoffmann, "Flow control of hypersonic separated flows by applied magnetic fields," in 44th AIAA Aerospace Sciences Meeting and Exhibit, Reno, Nevada, January 2006.
[9] W. Y. Su, X. Y. Chang, and K. Y. Zhang, "Effects of magnetohydrodynamic interaction-zone position on shock-wave/boundary-layer interaction," Journal of Propulsion and Power, vol. 26, no. 5, pp. 1053-1058, 2010.

[10] M. Atkinson, J. Poggie, and J. Camberos, "Numerical investigation of shock-wave/boundary-layer interaction control using plasma actuators," in 49th AIAA Aerospace Sciences Meeting Including the New Horizons Forum and Aerospace Exposition, Orlando, Florida, January 2011.

[11] F. Cheng, X. Zhong, S. Gogineni, and R. L. Kimmel, "Effect of applied magnetic field on the instability of Mach 4.5 boundary layer over a flat plate," in 40th AIAA Aerospace Sciences Meeting \& Exhibit, p. 351, Reno, Nevada, 2002.

[12] O. U. Khan, K. A. Hoffmann, and J. F. Dietiker, "Validity of low magnetic Reynolds number formulation of magnetofluiddynamic," AIAA Paper, vol. 2007-4374, 2007.

[13] G. A. Updike, J. S. Shang, and D. V. Gaitonde, "Hypersonic separated flow control using magneto-aerodynamic interaction," in 43rd AIAA Aerospace Sciences Meeting and Exhibit, Reno, Nevada, January 2005.

[14] N. Webb, C. Clifford, and M. Samimy, "Control of oblique shock wave-boundary layer interactions using plasma actuators," in 6th AIAA Flow Control Conference, New Orleans, Louisiana, June 2012.

[15] Q. Sun, Y. H. Li, W. Cui, B. Q. Cheng, J. Li, and H. Dai, "Shock wave-boundary layer interactions control by plasma aerodynamic actuation," Science China Technological Sciences, vol. 57, pp. 1335-1341, 2014.

[16] Y. Zhang, H. J. Tan, F. C. Tian, and Y. Zhuang, "Control of incident shock/boundary-layer interaction by a twodimensional bump," AIAA Journal, vol. 52, pp. 767-776, 2014.

[17] Z. A. Khan, S. U. Haq, T. S. Khan, I. Khan, and K. S. Nisar, "Fractional Brinkman type fluid in channel under the effect of MHD with Caputo-Fabrizio fractional derivative," Alexandria Engineering Journal, vol. 59, no. 5, pp. 2901-2910, 2020.

[18] T. Anwar, P. Kumam, Asifa, I. Khan, and P. Thounthong, "Generalized unsteady MHD natural convective flow of Jeffery model with ramped wall velocity and Newtonian heating; a Caputo-Fabrizio approach," Chinese Journal of Physics, vol. 68, pp. 849-865, 2020.

[19] K. Li, Mechanism Analysis of Magnetohydrodynamic Heat Shield System Including High Temperature Real Gas Effect [Ph.D. Thesis], National University of Defense Technology, 2017.

[20] J. Liu, Experimental and Numerical Research on ThermoChemical Nonequilibrium Flow with Radiation Phenomenon [Ph.D. Thesis], National University of Defense Technology, 2004.

[21] K. Li and W. Q. Liu, "Analysis of the magnetohydrodynamic heat shield system for hypersonic vehicles," Acta Physica Sinica, vol. 65, article 064701, 2016(in Chinese).

[22] K. Li, J. Liu, and W. Q. Liu, "Mechanism analysis of magnetohydrodynamic heat shield system and optimization of externally applied magnetic field," Acta Astronautica, vol. 133, pp. 14-23, 2017.

[23] X. M. Zheng, H. Y. Lu, D. J. Xu, and G. B. Cai, "Parameter research of an MHD controlled inlet," in 45th AIAA/ASME/SAE/ASEE Joint Propulsion Conference \& Exhibit, Denver, Colorado, August 2009.

[24] C. M. Hung and R. W. MacCormackt, "Numerical Solutions of Supersonic and Hypersonic Laminar Compression Corner Flows," AIAA Journal, vol. 14, no. 4, pp. 475-481, 1976. 
[25] D. Jean-Francois and H. Klaus, "A, modified one-equation turbulence models for turbulent magnetohydrodynamic flows," Journal of Thermophysics and Heat Transfer, vol. 17, no. 4, pp. 509-520, 2003.

[26] S. Saito, K. Udagawa, and K. Kawaguchi, "Boundary layer separation control by MHD interaction," in 46th AIAA Aerospace Sciences Meeting and Exhibit, Reno, Nevada, January 2008. 